\title{
Cognitive impairment in alcoholism
} Ilia Theotoka*

Address: Department of Psychiatry, Eginition Hospital, Athens University Medical School, Athens Greece

* Corresponding author

from International Society on Brain and Behaviour: 2nd International Congress on Brain and Behaviour

Thessaloniki, Greece. 17-20 November 2005

Published: 28 February 2006

Annals of General Psychiatry 2006, 5(SuppI I):S56 doi:10.1 186/1744-859X-5-SI-S56

The aim of this presentation is to describe the various cognitive domains affected in alcoholism and in alcohol related dementia. Numerous studies have shown the deterioration of cognitive function ranging from amnestic disorder to mild cognitive impairment, which is a syndrome between normal aging and early dementia, and to dementia. Nevertheless, it is unclear whether such cognitive deficits are reversible with abstinence. An association between alcohol abuse and cognitive deficits was observed in many studies. Some studies found that frontal lobe dysfunction (58\%) and short-term memory loss (32\%) were the most frequent cognitive abnormalities. Dementia was relatively uncommon (4\%). Episodic memory, abstraction, problem-solving and perceptual motor abilities were affected as well. Consolidation and deficits in recall have been also observed. In other studies deficits were disclosed in context and working memory, which were further supported by electrophysiological evidence of working memory deficits.

Memory and its subsystems (episodic, semantic, impliat memory) Language (naming, verbal fluency) visuoconstructive and visuospatial abilities, attention and executive functions will be examined through neuropsychological instruments, such as MMSE, Verbal Fluency Test, Clock Test, Wisconsin Card Sorting Test, subtests of WAIS. An emphasis will be given in executive dysfunction, which is more prominent in alcoholic patients.

In this presentation we will try also to investigate the spectrum of cognitive impairment that may be connected to alcohol related dementia, which is an entity with no standard diagnostic criteria.

We will describe also a neuropsychological study concerning cognitive deficits in alcoholic patients and patients with Alzheimer's disease. We evaluated the pattern of neuropsychological characteristics of 41 alcohol-dependent subjects (ALD) that reported memory disturbances, and compared it to the neuropsychological performance of 54 patients with mild-stage Alzheimer's disease (AD). Chronic ALD subjects had statistically significant higher scores on the Mini Mental State Examination (MMSE), the Syndrome Short Test (SKT), the Clock test, the Verbal Fluency Test Category, the Digit Span Forward Test, the Digit Span Backward Test ( $\mathrm{p}<0.005$, t-test) and higher scores on SKT1-5 and SKT7-9 (p < 0.005, Kruskal-Wallis test) compared to patients with AD. The mild cognitive deficit observed in ALD subjects does not involve memory tests, contrary to AD patients. Thus, it appears that the neuropsychological profile of ALD subjects is distinct from that of $\mathrm{AD}$ patients. Regarding clinical practice this distinction should be taken into consideration when alcoholic subjects are evaluated for the concomitant presence of $\mathrm{AD}$. 